\title{
Effect of Hexavalent Chromium on Electron Leakage of Respiratory Chain in Mitochondria Isolated from Rat Liver
}

\author{
Ying Xie Caigao Zhong Ming Zeng Lan Guan Lei Luo \\ Department of Health Toxicology, School of Public Health, Central South University, Changsha
}

\author{
Key Words \\ Hepatocyte $\cdot$ Mitochondria $•$ Electron leak $•$ GSH
}

\begin{abstract}
Background/Aims: In the present study, we explored reactive axygen species (ROS) production in mitochondria, the mechanism of hexavalent chromium $(\mathrm{Cr}(\mathrm{VI}))$ hepatotoxicity, and the role of protection by GSH. Methods: Intact mitochondria were isolated from rat liver tissues and mitochondrial basal respiratory rates of $\mathrm{NADH}$ and $\mathrm{FADH}_{2}$ respiratory chains were determined. Mitochondria were treated with $\mathrm{Cr}(\mathrm{VI}), \mathrm{GSH}$ and several complex inhibitors. Mitochondria energized by glutamate/malate were separately or jointly treated with Rotenone (Rot), diphenyleneiodonium (DPI) and antimycinA (Ant), while mitochondria energized by succinate were separately or jointly treated with Rot, DPI, thenoyltrifluoroacetone (TTFA) and Ant. Results: $\mathrm{Cr}(\mathrm{VI})$ concentration-dependently induced ROS production in the NADH and $\mathrm{FADH}_{2}$ respiratory chain in liver mitochondria. Basal respiratory rate of the mitochondrial FADH $\mathrm{H}_{2}$ respiratory chain was significantly higher than that of NADH respiratory chain. Hepatic mitochondrial electron leakage induced by $\mathrm{Cr}(\mathrm{VI})$ from NADH respiratory chain were mainly from ubiquinone binding sites of complex I and complex III. Conclusion: Treatment with 50 $\mathrm{MM}$ $\mathrm{Cr}(\mathrm{VI})$ enhances forward movement of electrons through $\mathrm{FADH}_{2}$ respiratory chain and leaking through the ubiquinone binding site of complex III. Moreover, the protective effect of GSH on liver mitochondria electron leakage is through removing excess $\mathrm{H}_{2} \mathrm{O}_{2}$ and reducing total ROS.
\end{abstract}

Copyright $@ 2013$ S. Karger AG, Basel

\section{Introduction}

Hexavalent chromium (Cr(VI)) is a well-known highly toxic metal and environmental pollutant, and was categorized as human carcinogen by IARC (International Agency for 


\section{Cellular Physiology and Biochemistry}

Cell Physiol Biochem 2013;31:473-485

\begin{tabular}{l|l}
\hline DOI: $10.1159 / 000350062$ & (c) 2013 S. Karger AG, Basel
\end{tabular}

Published onIIne: IVIarch 20, 2013

www.karger.com/cpb

Xie/Zhong/Zeng/Guan/Luo: Effect of Hexavalent Chromium on Electron Leakage
474

Research on Cancer) in 1990. Industrial sources of $\mathrm{Cr}(\mathrm{VI})$ include plating, electroplating, leather tanning, cooling tower blowdown, and anodizing baths, etc [1]. It has been reported that $\mathrm{Cr}(\mathrm{VI})$ would cause hepatocyte apoptosis, characterized by reactive oxygen species (ROS) generation [2]. Excess reactive oxygen species (ROS) production is the primary cause of mitochondrial function damage and energy metabolism disorder, leading to hepatocyte apoptosis.

Generally, the possible sources of ROS production is the mitochondrial electron transport chain in hepatocyte $[3,4]$. When exogenous toxins attack the mitochondrial respiratory chain, they would selectively inhibit mitochondrial complexes, resulting in blockage of normal electron transport passway and electron accumulation at the ubiquinone site. This would cause electron leakage from specific sites [5]. $\mathrm{O}_{2}$ would be reduced to superoxide anion radical $\left(\mathrm{O}_{2}^{--}\right)$by electron leaked from mitochondrial respiratory chain, which was further reduced to hydrogen peroxide $\left(\mathrm{H}_{2} \mathrm{O}_{2}\right)$ by superoxide dismutase (MnSOD) [4]. Then $\mathrm{H}_{2} \mathrm{O}_{2}$ would generate hydroxy radical $(\bullet \mathrm{OH})$ by reduction reaction. $\mathrm{O}_{2}{ }^{-}, \mathrm{H}_{2} \mathrm{O}_{2}$ and $\bullet \mathrm{OH}$ are referred as ROS, collectively.

Mitochondria contain several ROS production sites. Determination of the production site is based on using inhibitors of electron transport. For example, if rotenone (complex I inhibitor) could increase ROS production in cells, endogenous ROS production would be from complex I. Moreover, it is well known that GSH in liver mitochondria (mtGSH) plays an important role in clearing away $\mathrm{H}_{2} \mathrm{O}_{2}$ and preventing hepatocyte mitochondria from impairment of respiratory function and dysfunction of energy metabolism [6, 7]. Mitochondria electron transport chain and the role of GSH in scavenging ROS are illustrated in Figure 1.

Previous studies strongly suggest that ROS produced in mitochondria plays a critical role in $\mathrm{Cr}(\mathrm{VI})$-induced hepatocyte apoptosis. However, electron leakage of mitochondrial respiratory chain induced by $\mathrm{Cr}(\mathrm{VI})$ still remains unclear. In the present study, after treating isolated rat mitochondria with $\mathrm{Cr}(\mathrm{VI})$, we used glutamate/malate and succinate as substrates and a wide variety of complex inhibitors to explore the ROS production sites in mitochondria, the mechanism of $\mathrm{Cr}(\mathrm{VI})$ hepatotoxicity, and the role of GSH protection.

\section{Materials and Methods}

\section{Materials}

Rotenone (Rot), diphenyleneiodonium (DPI), thenoyltrifluoroacetone (TTFA), antimycinA (Ant), L-glutamine, L-Malic acid and sodium succinate were purchased from Sigma (St. Louis, MO, USA).

\section{Mitochondrial isolation}

Rats received anesthesia by chloralic hydras after an overnight fast. The rat liver was removed for immediate mitochondrial isolation. Liver mitochondria were isolated by standard differential centrifugation at $4^{\circ} \mathrm{C}$ as previously described with modifications [8]. Liver excised from rat were weighed and carefully homogenated in isolation buffer $(70 \mathrm{mM}$ sucrose, $210 \mathrm{mM}$ mannitol, 5mM Hepes, 1mM EGTA, 1\%BSA, $\mathrm{PH}=7.4$ ). Tissue debris and nuclei were separated from liver homogenate by centrifugation at $1000 \times \mathrm{g}$ for $10 \mathrm{~min}$. The supernatant solution was collected and centrifuged at $9000 \times \mathrm{g}$ for $10 \mathrm{~min}$. The mitochondria pellet was washed once with isolation buffer and re-suspended in respiratory buffer as mitochondria suspension (75mM sucrose, $50 \mathrm{mM} \mathrm{Kcl}, 30 \mathrm{mM}$ Tris- $\mathrm{Hcl}, 2 \mathrm{mM} \mathrm{KH}_{2} \mathrm{PO}_{4}, 2 \mathrm{mM} \mathrm{MgCl}_{2}, 10 \mu \mathrm{M}$ EGTA, PH=7.4). Isolated mitochondria were used immediately for measurement of respiration rate and ROS and superoxide formation.

\section{Mitochondrial protein measurements}

Mitochondrial protein concentrations were measured using a BCA protein quantification kit (Abcam, SF, USA) with bovine serum albumin used as concentration standards. Standards and the samples were measured in duplicates and the mean values were used to calculate the protein concentrations. 


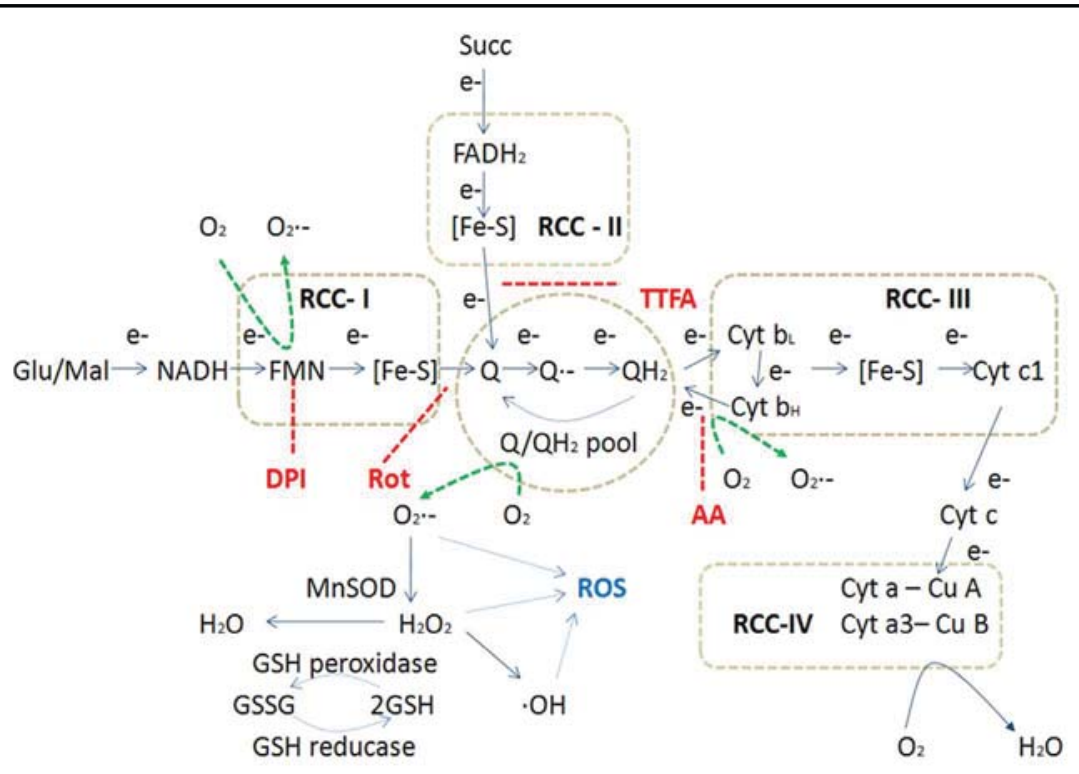

Fig. 1. Electron transport illustration chart of respiratory chain of hepatocyte mitochondria.

\section{Measurement of mitochondrial purity and functionality}

Mitochondria re-suspended in respiratory buffer were stored on the ice for 2.5 hours, then we measured purity and functionality of mitochondria isolated with our method. We detected mitochondrial purity by detection of succinate dehydrogenase (SDH) activity in liver homogenate, supernatant solution and mitochondria suspension, respectively with SDH kit (BIOTANG Inc., Waltham, MA 02452 USA). The mitochondria suspension were fixed overnight with $2.5 \%$ glutaraldehyde, postfixed with $2 \%$ osmid acid, dehydrated, and embedded with epoxy resin. Thin sections were observed with a Hitachi H-600 transmission electron microscope. Mitochondrial ultra-structure was observed by electron microscope (magnification $\times 10,000$ and $\times 30000$, respectively). Then respiratory function of mitochondria was measured with Clark-type oxygen electrode. One mg of mitochondria protein was incubated in $2 \mathrm{ml}$ of respiratory buffer preheated to $25^{\circ} \mathrm{C}$. After $2 \mathrm{~min}$ later, $5 \mathrm{~mm}$ succinate was added as substrate. Then $3 \mathrm{~min}$ later, state 3 respiration was initiated by adding $2 \mathrm{um} \mathrm{ADP}$, and oxygen consumption was measured as the slope of oxygen consumption curves. The respiratory control ratio (RCR) were calculated as the rate of ADP-stimulated oxygen consumption (State 3 respiratory) divided by the rate of oxygen consumption (state 4 respiratory) when all the ADP has been phosphorylated.

\section{Measurement of mitochondrial basal respiratory rate}

Mitochondrial basal respiratory rate of the $\mathrm{NADH}$ and $\mathrm{FADH}_{2}$ respiratory chain were measured using substrate-stimulated $\mathrm{O}_{2}$ consumption recorded with Clark-type oxygen electrode. Mitochondrial suspensions ( $1 \mathrm{mg}$ of protein) were diluted to a total volume of $2 \mathrm{ml}$ in reaction buffer preheated to $25^{\circ} \mathrm{C}$. Then $10 \mathrm{mM}$ glutamate $/ 5 \mathrm{mM}$ malate or $5 \mathrm{mM}$ succinate were used as substrate 2 min later, respectively. Respiration rate was measured by the slope of oxygen consumption curves after substrates were added as $\mathrm{nmol} \mathrm{O}_{2} / \mathrm{min} / \mathrm{mg}$ prot.

\section{Measurement of ROS formation}

Mitochondria $(0.5 \mathrm{mg} / \mathrm{ml})$ were energized with complex I $(10 \mathrm{mM}$ glutamate/ $5 \mathrm{mM}$ malate $)$ and complex II (5mM succinate) substrates respectively. Mitochondria suspension were treated with $\operatorname{Cr}(\mathrm{VI})$ at $0 \mu \mathrm{M}, 12.5 \mu \mathrm{M}, 25 \mu \mathrm{M}, 50 \mu \mathrm{M}$, and $100 \mu \mathrm{M}$. Liver mitochondria exhibited a concentration-dependent increase in ROS production. Considering feasible mitochondria activity and distinct ROS production, we choose $50 \mu \mathrm{M}$ $\mathrm{Cr}(\mathrm{VI})$ as the final experimental concentration. ROS production in mitochondria co-incubated with $50 \mu \mathrm{M}$ $\mathrm{Cr}(\mathrm{VI})$ was measured with addition of specific inhibitors of respiratory complexes and $200 \mu \mathrm{M}$ glutathione as the following: Complex I inhibitors, $6.35 \mu \mathrm{M}$ Rot, $10 \mu \mathrm{M}$ DPI; Complex II inhibitor, $20 \mu \mathrm{M}$ TTFA; Complex III inhibitor, $3.75 \mu \mathrm{M}$ Ant. ROS production was assessed with $10 \mu \mathrm{M} 2$ ',7'-dichlorfluorescein-diacetate 


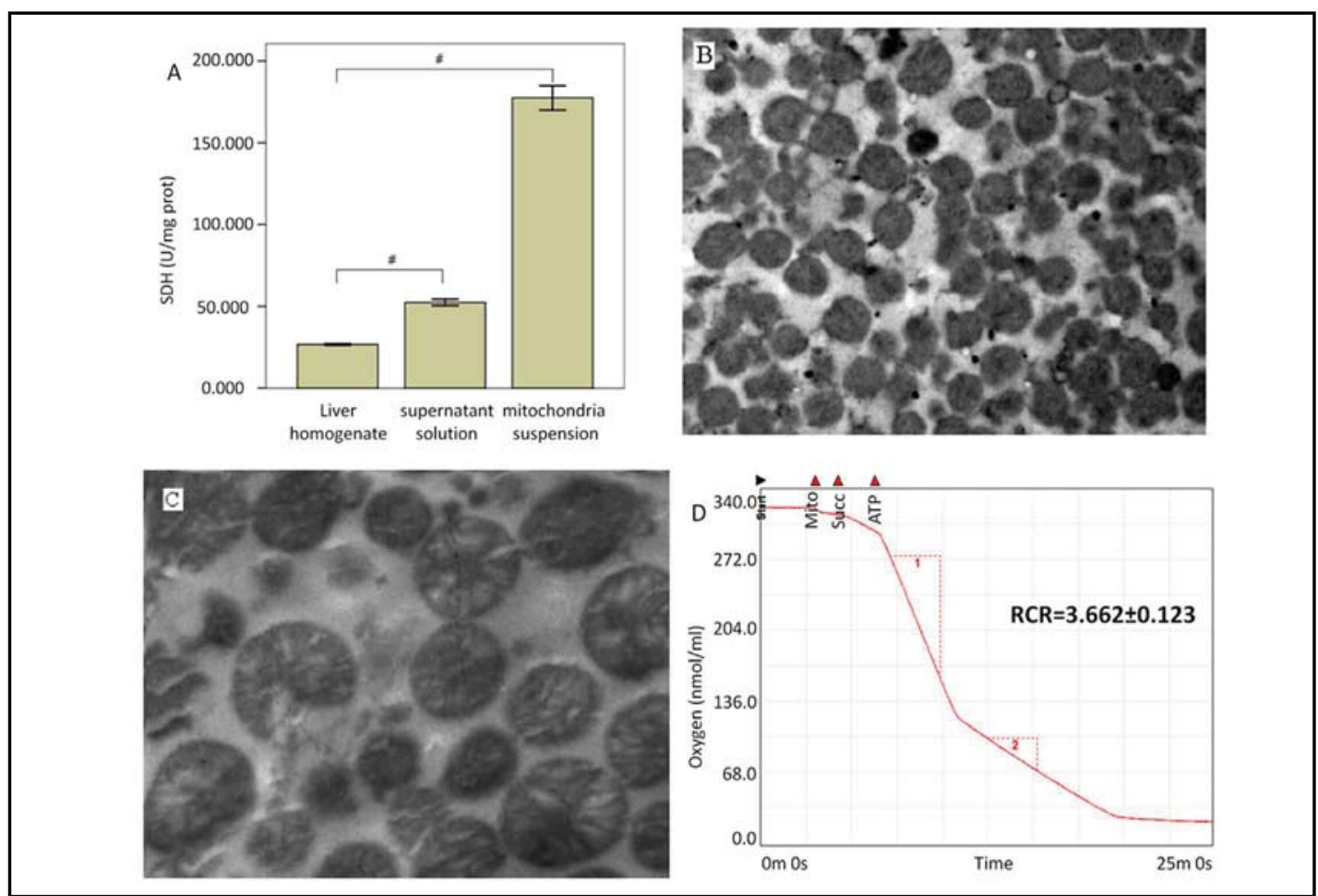

Fig. 2. Mitochondria purity and functionality. (A) Liver homogenate, supernatant solution and mitochondria suspension were analyzed for succinate dehydrogenase (SDH) activity. ${ }^{\#} \mathrm{P}<0.05$ vs liver homogenate group, $n=6$. Mitochondrial ultra-structure was observed by electron microscope at magnification $\times 10000$ (B) and x30000 (C), respectively. (D) Mitochondrial oxygen consumption curves. RCR is the ratio between the oxygen consumption in state 3 respiration (in the presence of ADP) and state 4 respiration (when all the ADP has been phosphorylated).

(DCFH-DA) fluorescent probes using VarioskanFlash 4.00.52 multifunctional microplate reader (excitation/ emission wavelengths: $488 \mathrm{~nm} / 525 \mathrm{~nm}$ ).

\section{Measurement of $\mathrm{O}_{2}^{--}$production and rate of electron leakage}

Mitochondria were used under similar experimental conditions as mentioned above to measure $\mathrm{O}_{2}{ }^{--}$ production using $30 \mu \mathrm{M}$ dihydroethidium (DHE) fluorescent probes. $\mathrm{O}_{2}{ }^{--}$-mediated formation of fluorescent ethidium was recorded at $\mathrm{Ex}_{498 \mathrm{~nm}} / \mathrm{Em}_{598 \mathrm{~nm}}$. The rate of electron leakage could be calculated as the rate of $\mathrm{O}_{2}^{--}$production $/ 2 \times$ rate of $\mathrm{O}_{2}$ consumption [9].

\section{Statistical analysis}

All data was expressed as mean \pm SEM. Statistical comparisons of basal respiratory rates of the mitochondrial respiratory chain were done using $t$ test. Comparisons among multiple treatments were done with analysis of variance (ANOVA). $P<0.05$ was considered statistically significant.

\section{Results}

Mitochondrial purity and functionality

Succinate hydrogenase is tightly bound to the inner mitochondrial membrane and easily precipitated along with mitochondria. The activities of succinate dehydrogenase as marker enzymes of mitochondria, in mitochondria suspension were significantly higher than that in liver homogenate (Fig. 2A), which revealed that mitochondria were relatively pure. 

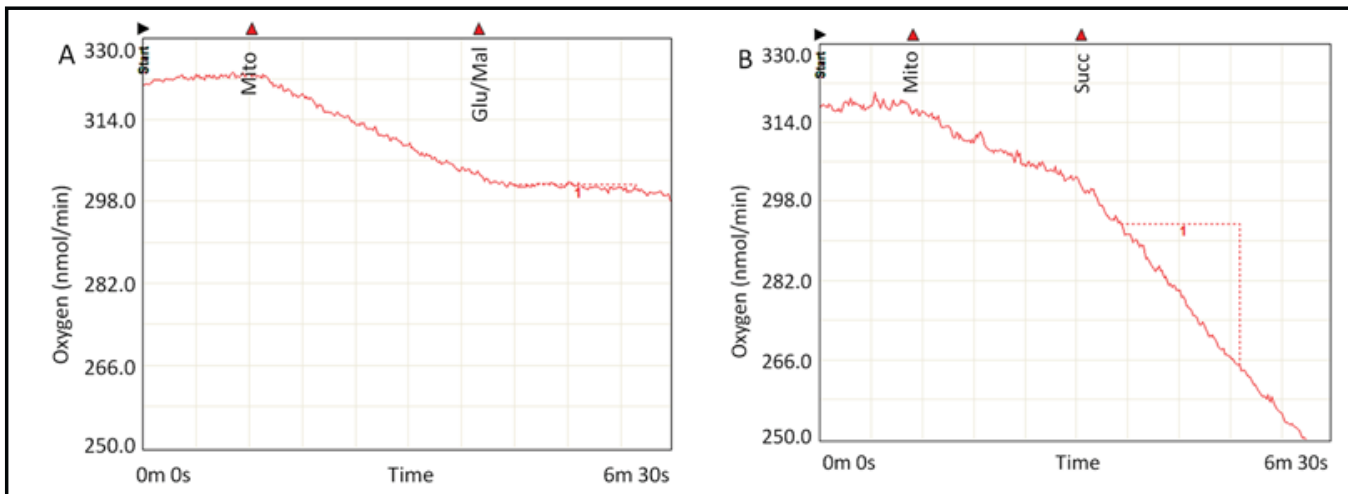

Fig. 3. Basal respiratory curves of mitochondrial $\mathrm{NADH}$ and $\mathrm{FADH}_{2}$ respiratory chain. (A) Basal respiratory curves of mitochondrial NADH respiratory chain; (B) Basal respiratory curves of mitochondrial $\mathrm{FADH}_{2}$ respiratory chain; (C) Comparison of basal respiratory rates between mitochondrial NADH and $\mathrm{FADH}_{2}$ respiratory chain. ${ }^{\#} \mathrm{P}<0.05$ vs NADH respiratory chain, $\mathrm{n}=6$.

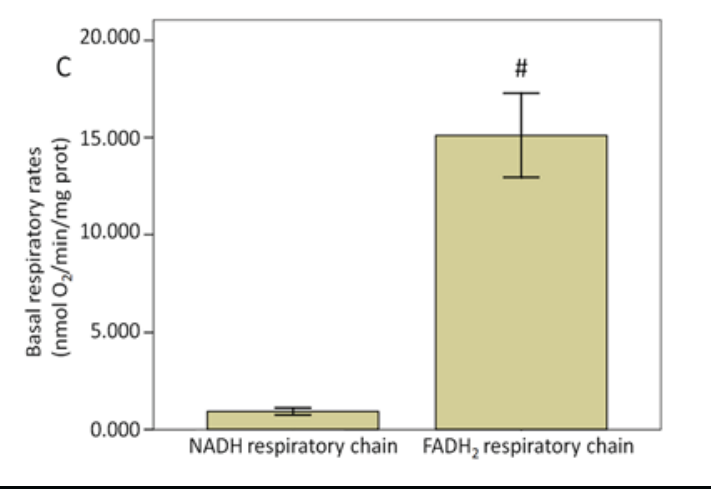

Table 1. Basal respiratory rate of mitochondrial $\mathrm{NADH}$ and $\mathrm{FADH}_{2}$ respiratory chain. Note: ${ }^{\#} P<0.05$ vs NADH respiratory chain

\begin{tabular}{ccccc}
\hline Groups & $\mathrm{N}$ & $\begin{array}{c}\text { Basal respiratory rate } \\
\left(\mathrm{nmol} \mathrm{O}_{2} / \mathrm{min} / \mathrm{mgprot}\right)\end{array}$ & $\mathrm{t}$ & $P$ \\
\hline NADH respiratory chain & 6 & $0.935 \pm 0.170$ & -0.642 & 0.001 \\
\hline FADH $\mathrm{F}_{2}$ respiratory chain & 6 & $15.350 \pm 0.689^{\#}$ & & \\
\hline
\end{tabular}

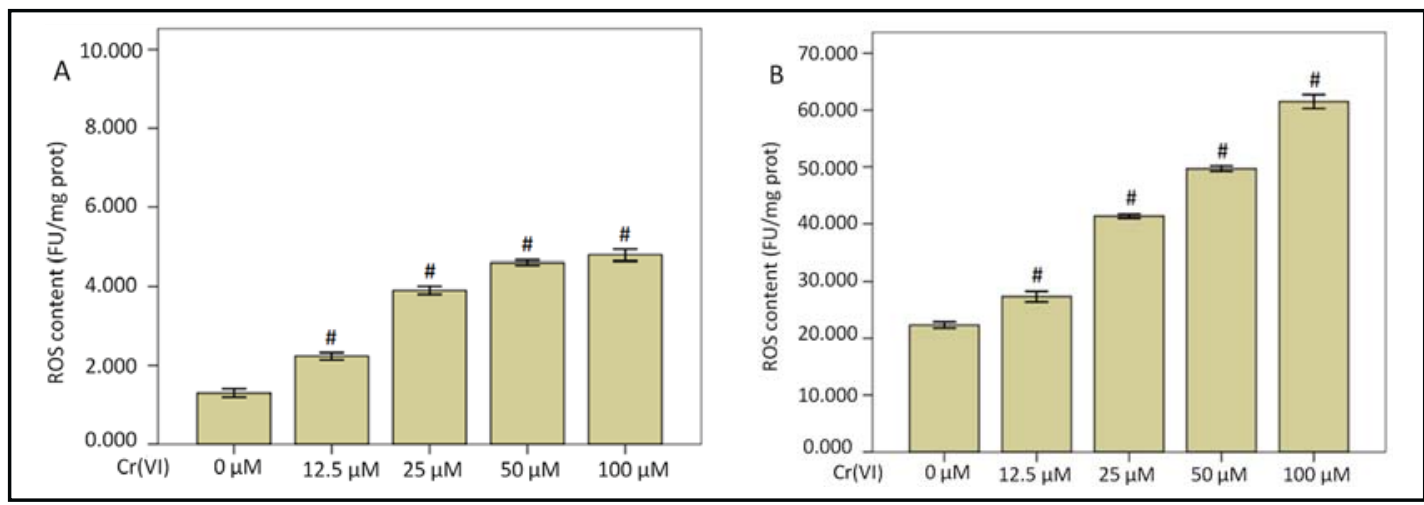

Fig. 4. ROS content of isolated rat mitochondria exposed to $\mathrm{Cr}(\mathrm{VI})$. (A) ROS production of mitochondrial NADH respiratory chain induced by $\mathrm{Cr}(\mathrm{VI})$; (B) ROS production of mitochondrial $\mathrm{FADH}_{2}$ respiratory chain induced by $\mathrm{Cr}(\mathrm{VI})$. ${ }^{*} \mathrm{P}<0.05$ vs $0 \mu \mathrm{m} \mathrm{Cr}(\mathrm{VI})$ group, $\mathrm{n}=6$.

Mitochondrial ultra-structure observation showed that mitochondria were relatively pure and morphologically intact and dense with no signs of swelling and damage (Fig. 2B, 2C). Mitochondrial respiratory control ratios (RCR) were higher than 3, which was indicative of tightly coupled mitochondria (Fig. 2D) [10]. 
Fig. 5. Effect of complex I inhibitors and complex III inhibitors on NADH respiratory chain in liver mitochondria. (A)(B) Effect of complex I inhibitors (ROT and DPI) on ROS production and electron leak rate from mitochondrial NADH respiratory chain induced by Cr(VI); (C)(D) Effect of complex III inhibitors (Ant) on ROS production and electron leak rate from mitochondrial NADH respiratory chain induced by $\mathrm{Cr}(\mathrm{VI})$. The concentrations of $\mathrm{Cr}(\mathrm{VI})$, Rot, DPI and Ant were $50 \mu \mathrm{M}, 6.35 \mu \mathrm{M}, 10 \mu \mathrm{M}$ and $3.75 \mu \mathrm{M}$, respectively. ${ }^{\#} \mathrm{P}<0.05, \mathrm{n}=6$.
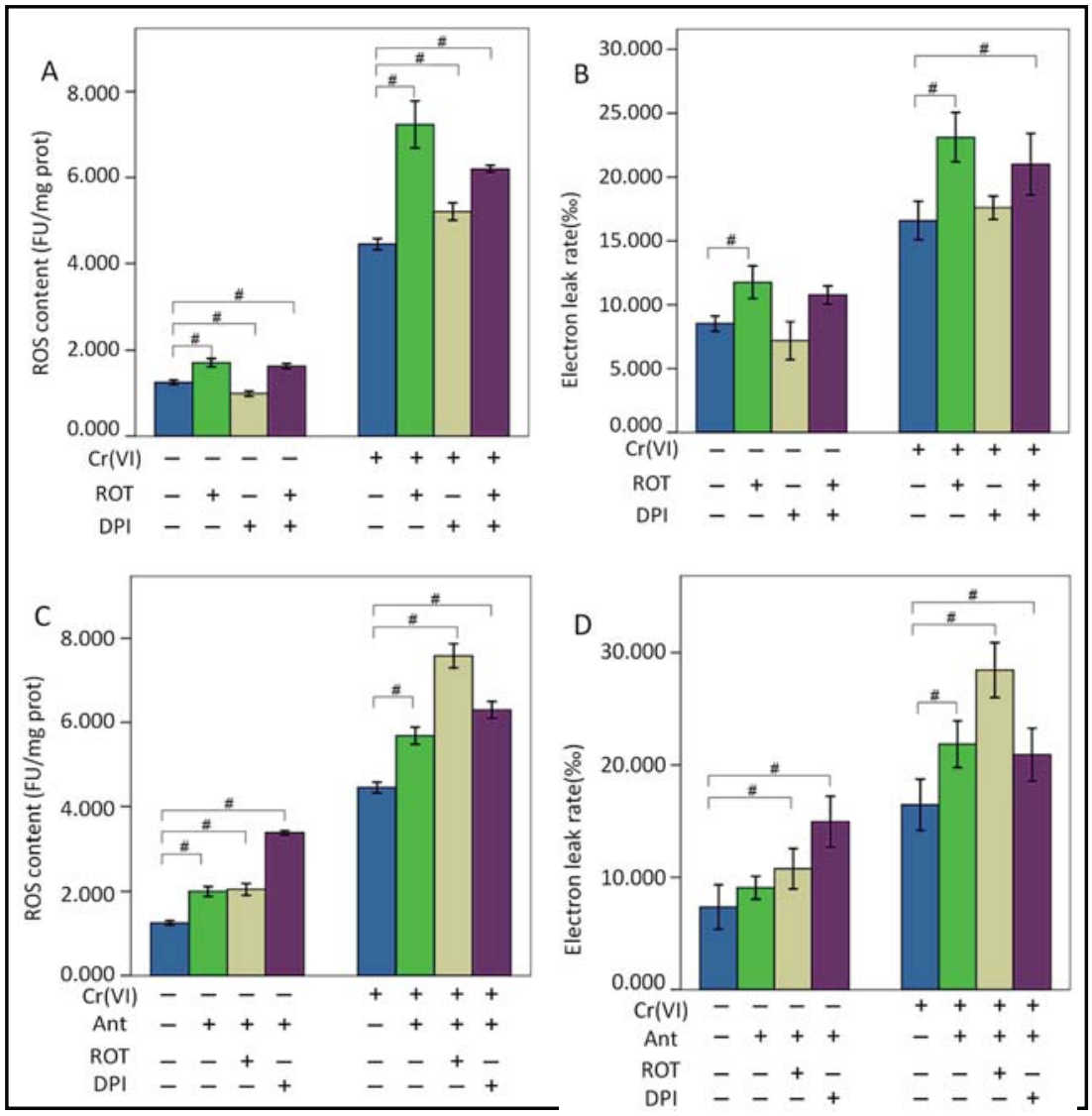

\begin{tabular}{cccccc}
\hline Groups & $\mathrm{N}$ & $\begin{array}{c}\text { ROS content } \\
(\mathrm{FU} / \mathrm{mgprot})\end{array}$ & $\begin{array}{c}\mathrm{O}_{2}^{*-} \text { content } \\
(\mathrm{FU} / \mathrm{mgprot})\end{array}$ & $\begin{array}{c}\mathrm{O}_{2}{ }^{*} \text {-content } \\
(\mathrm{nmol} / \mathrm{min} / \mathrm{mgprot})\end{array}$ & $\begin{array}{c}\text { electron leak rate } \\
(\% \mathrm{\%})\end{array}$ \\
\hline control & 6 & $1.252 \pm 0.047$ & $0.431 \pm 0.097$ & $0.137 \pm 0.035$ & $7.352 \pm 1.891$ \\
Rot & 6 & $1.709 \pm 0.095^{*}$ & $0.623 \pm 0.191^{\#}$ & $0.208 \pm 0.070^{\#}$ & $11.100 \pm 3.749^{\#}$ \\
DPI & 6 & $0.991 \pm 0.054^{\#}$ & $0.379 \pm 0.084$ & $0.118 \pm 0.031$ & $6.320 \pm 1.643$ \\
Rot+DPI & 6 & $1.627 \pm 0.057^{\#}$ & $0.591 \pm 0.129$ & $0.196 \pm 0.047$ & $10.486 \pm 2.521$ \\
Cr(VI) & 6 & $4.452 \pm 0.119^{*}$ & $0.885 \pm 0.315$ & $0.304 \pm 0.115$ & $16.232 \pm 6.169$ \\
Cr(VI)+Rot & 6 & $7.237 \pm 0.525^{*}$ & $1.236 \pm 0.094^{*}$ & $0.432 \pm 0.034^{*}$ & $23.114 \pm 1.843^{*}$ \\
Cr(VI)+DPI & 6 & $5.205 \pm 0.192^{*}$ & $0.955 \pm 0.045$ & $0.329 \pm 0.016$ & $17.603 \pm 0.873$ \\
Cr(VI)+Rot+DPI & 6 & $6.195 \pm 0.078^{*}$ & $1.121 \pm 0.137^{*}$ & $0.390 \pm 0.050^{*}$ & $20.868 \pm 2.690^{*}$ \\
\hline
\end{tabular}

Table 2. Effect of complex I inhibitors on NADH respiratory chain in liver mitochondria. Note: ${ }^{\#}<0.05$ vs the control group; ${ }^{*} P<0.05$ vs the $\mathrm{Cr}(\mathrm{VI})$ group. The concentrations of $\mathrm{Cr}(\mathrm{VI})$, Rot, DPI were $50 \mu \mathrm{M}, 6.35 \mu \mathrm{M}$ and $10 \mu \mathrm{M}$, respectively

\section{Basal respiration rate of $\mathrm{NADH}$ and $\mathrm{FADH}_{2}$ respiratory chain}

$\mathrm{O}_{2}$ consumption of glutamate/malate-energized and succinate-energized liver mitochondria was measured as basal respiratory rate of $\mathrm{NADH}$ and $\mathrm{FADH}_{2}$ respiratory chain (Fig. 3A, 3B). The basal respiratory rate of $\mathrm{FADH}_{2}$ respiratory chain was much greater than that of NADH respiratory chain (Fig. 3C, Table 1).

ROS production of mitochondria induced by $\operatorname{Cr}(V I)$ of different concentrations

$\mathrm{Cr}(\mathrm{VI})$ could induce the increase of ROS production in NADH and $\mathrm{FADH}_{2}$ respiratory chain in liver mitochondria in a concentration-dependent manner (Fig. 4). 


\begin{tabular}{cccccc}
\hline Groups & $\mathrm{N}$ & $\begin{array}{c}\mathrm{ROS} \text { content } \\
\text { (FU/mgprot) }\end{array}$ & $\begin{array}{c}\mathrm{O}_{2}{ }^{*-} \text { content } \\
\text { (FU/mgprot) }\end{array}$ & $\begin{array}{c}\mathrm{O}_{2}{ }^{*-} \text { production rate } \\
(\mathrm{nmol} / \mathrm{min} / \mathrm{mgprot})\end{array}$ & $\begin{array}{c}\text { electron leak rate } \\
(\% \mathrm{~m})\end{array}$ \\
\hline control & 6 & $1.252 \pm 0.047$ & $0.431 \pm 0.097$ & $0.137 \pm 0.035$ & $7.352 \pm 1.891$ \\
Ant & 6 & $2.002 \pm 0.111^{\#}$ & $0.519 \pm 0.506$ & $0.169 \pm 0.019$ & $9.062 \pm 0.991$ \\
Rot+Ant & 6 & $2.053 \pm 0.135^{\#}$ & $0.605 \pm 0.087^{\#}$ & $0.201 \pm 0.032^{\#}$ & $10.760 \pm 1.713^{\#}$ \\
DPI+Ant & 6 & $3.378 \pm 0.049^{\#}$ & $0.819 \pm 0.111^{\#}$ & $0.279 \pm 0.041^{\#}$ & $14.939 \pm 2.166^{\#}$ \\
Cr(VI) & 6 & $4.452 \pm 0.119$ & $0.885 \pm 0.315$ & $0.304 \pm 0.115$ & $16.232 \pm 6.169$ \\
Cr(VI)+Ant & 6 & $5.684 \pm 0.192^{*}$ & $1.172 \pm 0.101^{*}$ & $0.409 \pm 0.037^{*}$ & $21.860 \pm 1.975^{*}$ \\
Cr(VI)+Rot+Ant & 6 & $7.580 \pm 0.272^{*}$ & $1.508 \pm 0.119^{*}$ & $0.532 \pm 0.043^{*}$ & $28.442 \pm 2.324^{*}$ \\
Cr(VI)+DPI+Ant & 6 & $6.298 \pm 0.184^{*}$ & $1.124 \pm 0.115^{*}$ & $0.391 \pm 0.042^{*}$ & $20.920 \pm 2.249^{*}$ \\
\hline
\end{tabular}

Table 3. Effect of complex III inhibitors on NADH respiratory chain in liver mitochondria. Note: ${ }^{\#} P<0.05$ vs the control group; ${ }^{*} P<0.05$ vs the $\mathrm{Cr}(\mathrm{VI})$ group. The concentrations of $\mathrm{Cr}(\mathrm{VI})$, Rot, DPI and Ant were $50 \mu \mathrm{M}$, $6.35 \mu \mathrm{M}, 10 \mu \mathrm{M}$ and $3.75 \mu \mathrm{M}$, respectively.

\begin{tabular}{cccccc}
\hline Groups & $\mathrm{N}$ & $\begin{array}{c}\text { ROS content } \\
\text { (FU/mgprot) }\end{array}$ & $\begin{array}{c}\mathrm{O}_{2}{ }^{*-} \text { content } \\
(\mathrm{FU} / \mathrm{mgprot})\end{array}$ & $\begin{array}{c}\mathrm{O}_{2}{ }^{*-} \text { production rate } \\
(\mathrm{nmol} / \mathrm{min} / \mathrm{mgprot})\end{array}$ & $\begin{array}{c}\text { electron leak rate } \\
(\%)\end{array}$ \\
\hline control & 6 & $22.905 \pm 0.501$ & $0.699 \pm 0.021$ & $0.235 \pm 0.008$ & $0.767 \pm 0.025$ \\
Rot & 6 & $10.665 \pm 0.660 \#$ & $0.338 \pm 0.098 \#$ & $0.104 \pm 0.036 \#$ & $0.337 \pm 0.118 \#$ \\
DPI & 6 & $16.048 \pm 0.594^{\#}$ & $0.448 \pm 0.046 \#$ & $0.144 \pm 0.017 \#$ & $0.469 \pm 0.054 \#$ \\
Rot+DPI & 6 & $16.305 \pm 0.921^{\#}$ & $0.469 \pm 0.069 \#$ & $0.152 \pm 0.025^{\#}$ & $0.496 \pm 0.082^{\#}$ \\
Cr(VI) & 6 & $36.326 \pm 0.357$ & $1.006 \pm 0.065$ & $0.348 \pm 0.024$ & $1.134 \pm 0.078$ \\
Cr(VI)+Rot & 6 & $37.237 \pm 2.818$ & $1.059 \pm 0.107$ & $0.368 \pm 0.039$ & $1.197 \pm 0.128$ \\
Cr(VI)+DPI & 6 & $35.409 \pm 0.501$ & $1.083 \pm 0.051$ & $0.376 \pm 0.019$ & $1.225 \pm 0.061$ \\
Cr(VI)+Rot+DPI & 6 & $35.435 \pm 1.856$ & $1.104 \pm 0.095$ & $0.384 \pm 0.035$ & $1.251 \pm 0.113$ \\
\hline
\end{tabular}

Table 4. Effect of complex I inhibitors on $\mathrm{FADH}_{2}$ respiratory chain in liver mitochondria. Note: ${ }^{\#} P<0.05$ vs the control group; ${ }^{*} P<0.05$ vs the $\mathrm{Cr}(\mathrm{VI})$ group. The concentrations of $\mathrm{Cr}(\mathrm{VI})$, Rot, DPI were $50 \mu \mathrm{M}, 6.35 \mu \mathrm{M}$ and $10 \mu \mathrm{M}$, respectively.

\begin{tabular}{|c|c|c|c|c|c|}
\hline Groups & $\mathrm{N}$ & $\begin{array}{l}\text { ROS content } \\
\text { (FU/mgprot) }\end{array}$ & $\begin{array}{l}\mathrm{O}_{2} *-\text { content } \\
\text { (FU/mgprot) }\end{array}$ & $\begin{array}{l}\mathrm{O}_{2}{ }^{*-} \text { production rate } \\
\text { (nmol/min/mgprot) }\end{array}$ & $\begin{array}{c}\text { electron leak rate } \\
(\% 0)\end{array}$ \\
\hline control & 6 & $22.905 \pm 0.501$ & $0.699 \pm 0.021$ & $0.235 \pm 0.008$ & $0.767 \pm 0.025$ \\
\hline TTFA & 6 & $35.369 \pm 2.205$ \# & $1.155 \pm 0.079 \#$ & $0.403 \pm 0.029 \#$ & $1.312 \pm 0.094$ \# \\
\hline Rot+TTFA & 6 & $20.580 \pm 1.576 \#$ & $0.713 \pm 0.800$ & $0.241 \pm 0.029$ & $0.786 \pm 0.095$ \\
\hline DPI+TTFA & 6 & $16.134 \pm 0.447$ \# & $0.430 \pm 0.044 \#$ & $0.137 \pm 0.016 \#$ & $0.446 \pm 0.053 \#$ \\
\hline $\mathrm{Cr}(\mathrm{VI})$ & 6 & $36.326 \pm 0.357$ & $1.006 \pm 0.065$ & $0.348 \pm 0.024$ & $1.134 \pm 0.078$ \\
\hline $\mathrm{Cr}(\mathrm{VI})+\mathrm{TTFA}$ & 6 & $42.653 \pm 0.978^{*}$ & $1.456 \pm 0.128 *$ & $0.513 \pm 0.047^{*}$ & $1.671 \pm 0.153^{*}$ \\
\hline $\mathrm{Cr}(\mathrm{VI})+\mathrm{Rot}+\mathrm{TTFA}$ & 6 & $38.130 \pm 2.058^{*}$ & $1.215 \pm 0.064^{*}$ & $0.425 \pm 0.023^{*}$ & $1.382 \pm 0.076^{*}$ \\
\hline $\mathrm{Cr}(\mathrm{VI})+\mathrm{DPI}+\mathrm{TTFA}$ & 6 & $28.630 \pm 0.332^{*}$ & $0.787 \pm 0.061^{*}$ & $0.268 \pm 0.022^{*}$ & $0.873 \pm 0.072^{*}$ \\
\hline
\end{tabular}

Table 5. Effect of complex II inhibitors on $\mathrm{FADH}_{2}$ respiratory chain in liver mitochondria. Note: ${ }^{\sharp} P<0.05$ vs the control group; ${ }^{*} P<0.05$ vs the $\mathrm{Cr}(\mathrm{VI})$ group. The concentrations of $\mathrm{Cr}(\mathrm{VI})$, Rot, DPI and TTFA were $50 \mu \mathrm{M}$, $6.35 \mu \mathrm{M}, 10 \mu \mathrm{M}$ and $20 \mu \mathrm{M}$, respectively.

\section{Effect of complex inhibitors on NADH respiratory chain in liver mitochondria treated with $\mathrm{Cr}(\mathrm{VI})$}

In glutamate/malate-energized mitochondria, ROS production increased in the Rot group and the Rot+DPI group and decreased in the DPI group. In mitochondria treated with $\mathrm{Cr}(\mathrm{VI})$, ROS production increased significantly in the $\mathrm{Cr}(\mathrm{VI})+$ Rot group, the $\mathrm{Cr}(\mathrm{VI})+$ Rot+DPI group, and the $\mathrm{Cr}(\mathrm{VI})+\mathrm{DPI}$ group (Fig. 5A, 5B), Table 2. Ant could significantly increase ROS production and electron leakage in mitochondria treated with or without $\mathrm{Cr}$ (VI) (Fig .5C, 5D), Table 3. 



Fig. 6. Effect of complex I inhibitors and complex III inhibitors on $\mathrm{FADH}_{2}$ respiratory chain in liver mitochondria. (A) (B) Effect of complex I inhibitors (ROT and DPI) on ROS production and electron leak rate from mitochondrial $\mathrm{FADH}_{2}$ respiratory chain induced by $\mathrm{Cr}(\mathrm{VI})$; (C)(D) Effect of complex II inhibitors (TTFA) on ROS production and electron leak rate from mitochondrial $\mathrm{FADH}_{2}$ respiratory chain induced by $\mathrm{Cr}(\mathrm{VI})$; (E)(F) Effect of complex III inhibitors (Ant) on ROS production and electron leak rate from mitochondrial $\mathrm{FADH}_{2}$ respiratory chain induced by $\mathrm{Cr}(\mathrm{VI})$. The concentration of $\mathrm{Cr}(\mathrm{VI})$, Rot, DPI, TTFA and Ant were $50 \mu \mathrm{M}$, $6.35 \mu \mathrm{M}, 10 \mu \mathrm{M}, 20 \mu \mathrm{M}$ and $3.75 \mu \mathrm{M}$, respectively. ${ }^{\#} \mathrm{P}<0.05, \mathrm{n}=6$.

\begin{tabular}{cccccc}
\hline Groups & $\mathrm{N}$ & $\begin{array}{c}\text { ROS content } \\
\text { (FU/mgprot) }\end{array}$ & $\begin{array}{c}\mathrm{O}_{2}^{*-} \text { content } \\
\text { (FU/mgprot) }\end{array}$ & $\begin{array}{c}\mathrm{O}_{2}^{*-} \text { production rate } \\
(\mathrm{nmol} / \mathrm{min} / \mathrm{mgprot})\end{array}$ & $\begin{array}{c}\text { electron leak } \\
\text { rate }(\% \mathrm{o})\end{array}$ \\
\hline control & 6 & $22.905 \pm 0.501$ & $0.699 \pm 0.021$ & $0.235 \pm 0.008$ & $0.767 \pm 0.025$ \\
Ant & 6 & $29.240 \pm 2.519^{\#}$ & $1.003 \pm 0.081^{\#}$ & $0.347 \pm 0.030^{\#}$ & $1.130 \pm 0.096 \#$ \\
Rot+Ant & 6 & $30.497 \pm 1.463^{\#}$ & $1.074 \pm 0.197 \#$ & $0.374 \pm 0.072^{\#}$ & $1.217 \pm 0.235 \#$ \\
DPI+Ant & 6 & $28.991 \pm 1.388^{\#}$ & $0.781 \pm 0.102$ & $0.266 \pm 0.038$ & $0.866 \pm 0.122$ \\
TTFA+Ant & 6 & $37.030 \pm 0.888^{\#}$ & $1.126 \pm 0.150^{\#}$ & $0.392 \pm 0.055^{\#}$ & $1.277 \pm 0.179^{\#}$ \\
Cr(VI) & 6 & $36.326 \pm 0.357$ & $1.006 \pm 0.065^{\circ}$ & $0.348 \pm 0.024$ & $1.134 \pm 0.078$ \\
Cr(VI)+Ant & 6 & $53.031 \pm 2.265^{*}$ & $1.165 \pm 0.055^{*}$ & $0.407 \pm 0.020^{*}$ & $1.325 \pm 0.065^{*}$ \\
Cr(VI)+Rot+Ant & 6 & $42.818 \pm 0.694^{*}$ & $1.557 \pm 0.061^{*}$ & $0.550 \pm 0.022^{*}$ & $1.791 \pm 0.072^{*}$ \\
Cr(VI)+DPI+Ant & 6 & $41.486 \pm 3.265^{*}$ & $1.059 \pm 0.082$ & $0.368 \pm 0.030$ & $1.197 \pm 0.098$ \\
Cr(VI)+TTFA+Ant & 6 & $47.715 \pm 2.015^{*}$ & $1.451 \pm 0.061^{*}$ & $0.511 \pm 0.025^{*}$ & $1.665 \pm 0.082^{*}$ \\
\hline
\end{tabular}

Table 6. Effect of complex III inhibitors on $\mathrm{FADH}_{2}$ respiratory chain in liver mitochondria. Note: ${ }^{\#} P<0.05$ vs the control group; ${ }^{*} P<0.05$ vs the $\mathrm{Cr}(\mathrm{VI})$ group. The concentration of $\mathrm{Cr}(\mathrm{VI})$, Rot, DPI , TTFA and Ant were $50 \mu \mathrm{M}, 6.35 \mu \mathrm{M}, 10 \mu \mathrm{M}, 20 \mu \mathrm{M}$ and $3.75 \mu \mathrm{M}$, respectively.

Effect of complex inhibitors on $\mathrm{FADH}_{2}$ respiratory chain in liver mitochondria treated with $\operatorname{Cr}(V I)$

In succinate-energized liver mitochondria, ROS production and electron leakage decreased significantly in the Rot group, the DPI group and the Rot+DPI group. However, in mitochondria treated with $\mathrm{Cr}(\mathrm{VI})$, no significant difference was observed among the 
Fig. 7. Effect of GSH on electron leakage from mitochondrial $\mathrm{NADH}$ and $\mathrm{FADH}_{2}$ respiratory chain induced by $\mathrm{Cr}(\mathrm{VI})$. (A)(B) Effect of GSH on ROS production and electron leakage from mitochondrial NADH respiratory chain induced by $\mathrm{Cr}(\mathrm{VI})$; (C)(D) Effect of GSH on ROS production and electron leakage from mitochondrial $\mathrm{FADH}_{2}$ respiratory chain induced by Cr(VI). The concentration of $\mathrm{Cr}(\mathrm{VI})$, GSH, Rot, DPI, TTFA and Ant were $50 \mu \mathrm{M}$, $200 \mu \mathrm{M}, \quad 6.35 \mu \mathrm{M}$, $10 \mu \mathrm{M}, \quad 20 \mu \mathrm{M}$ and $3.75 \mu \mathrm{M}$, respectively. ${ }^{\#} \mathrm{P}<0.05, \mathrm{n}=6$.


\begin{tabular}{cccccc}
\hline \multirow{2}{*}{ Groups } & $\mathrm{N}$ & \multicolumn{2}{c}{$\begin{array}{c}\text { ROS content } \\
\text { (FU/mgprot) }\end{array}$} & \multicolumn{2}{c}{$\begin{array}{c}\text { electron leak rate } \\
\text { (\%o) }\end{array}$} \\
& & $\mathrm{Cr}(\mathrm{VI})$ & $\mathrm{Cr}(\mathrm{VI})+\mathrm{GSH}$ & $\mathrm{Cr}(\mathrm{VI})$ & $\mathrm{Cr}(\mathrm{VI})+\mathrm{GSH}$ \\
\hline control & 6 & $4.452 \pm 0.119$ & $1.712 \pm 0.060$ & $16.232 \pm 6.169$ & $14.900 \pm 8.440$ \\
Rot & 6 & $7.237 \pm 0.525$ & $5.057 \pm 0.072^{\#}$ & $23.114 \pm 1.843$ & $22.748 \pm 2.137$ \\
DPI & 6 & $5.205 \pm 0.192$ & $4.143 \pm 0.131 \#$ & $17.603 \pm 0.873$ & $18.269 \pm 2.582$ \\
Ant & 6 & $5.684 \pm 0.192$ & $4.285 \pm 0.122^{\#}$ & $21.860 \pm 1.975$ & $22.540 \pm 1.324$ \\
Rot+DPI & 6 & $6.195 \pm 0.078$ & $4.025 \pm 0.092^{\#}$ & $20.868 \pm 2.691$ & $21.051 \pm 2.593$ \\
Rot+Ant & 6 & $7.580 \pm 0.272$ & $5.866 \pm 0.163 \#$ & $28.442 \pm 2.324$ & $27.502 \pm 2.554$ \\
DPI+Ant & 6 & $6.298 \pm 0.184$ & $4.053 \pm 0.142^{\#}$ & $20.920 \pm 2.249$ & $20.528 \pm 2.742$ \\
\hline
\end{tabular}

Table 7. Effect of GSH on electron leakage from mitochondrial NADH respiratory chain induced by $\mathrm{Cr}$ (VI) Note: ${ }^{*} \mathrm{P}<0.05$ vs the $\mathrm{Cr}(\mathrm{VI})$ group. The concentration of $\mathrm{Cr}(\mathrm{VI}), \mathrm{GSH}, \mathrm{Rot}, \mathrm{DPI}$, TTFA and Ant were $50 \mu \mathrm{M}$, $200 \mu \mathrm{M}, 6.35 \mu \mathrm{M}, 10 \mu \mathrm{M}, 20 \mu \mathrm{M}$ and $3.75 \mu \mathrm{M}$, respectively.

$\mathrm{Cr}(\mathrm{VI})+$ Rot group, the $\mathrm{Cr}(\mathrm{VI})+\mathrm{DPI}$ group and the $\mathrm{Cr}(\mathrm{VI})+$ Rot+DPI group (Fig. 6A, 6B, Table 4). Then we investigated the effect of TTFA, specific inhibitor of complex II, on $\mathrm{FADH}_{2}$ respiratory chain. TTFA could increase ROS production and electron leakage in mitochondria treated with $\mathrm{Cr}(\mathrm{VI})$ with or without Rot, which was reversed by DPI (Fig. 6C, 6D, Table 5). The effect of Ant on FADH 2 respiratory chain was similar to that on NADH respiratory chain. ROS production and electron leakage in groups treated with Ant were higher than those not treated with Ant(Fig. 6E, 6F, Table 6). 


\section{Cellular Physiology and Biochemistry}

Cell Physiol Biochem 2013;31:473-485

\begin{tabular}{cccccc}
\hline Groups & N & \multicolumn{2}{c}{$\begin{array}{c}\text { ROS content } \\
\text { (FU/mgprot) }\end{array}$} & \multicolumn{2}{c}{$\begin{array}{c}\text { electron leak rate } \\
\text { (\%o) }\end{array}$} \\
& & $\mathrm{Cr}(\mathrm{VI})$ & $\mathrm{Cr}(\mathrm{VI})+\mathrm{GSH}$ & $\mathrm{Cr}(\mathrm{VI})$ & $\mathrm{Cr}(\mathrm{VI})+\mathrm{GSH}$ \\
\hline control & 6 & $36.326 \pm 0.357$ & $27.288 \pm 1.487 \#$ & $1.134 \pm 0.078$ & $1.204 \pm 0.033$ \\
Rot & 6 & $37.237 \pm 2.818$ & $30.909 \pm 0.695 \#$ & $1.197 \pm 0.128$ & $1.214 \pm 0.122$ \\
DPI & 6 & $35.409 \pm 0.501$ & $23.204 \pm 1.459 \#$ & $1.225 \pm 0.061$ & $1.299 \pm 0.173$ \\
TTFA & 6 & $42.653 \pm 0.978$ & $30.948 \pm 1.118^{\#}$ & $1.671 \pm 0.153$ & $1.708 \pm 0.068$ \\
Ant & 6 & $43.031 \pm 2.265$ & $17.411 \pm 0.473 \#$ & $1.325 \pm 0.065$ & $1.329 \pm 0.121$ \\
Rot+DPI & 6 & $35.435 \pm 1.856$ & $24.297 \pm 0.803^{\#}$ & $1.250 \pm 0.113$ & $1.336 \pm 0.166$ \\
Rot+TTFA & 6 & $38.130 \pm 2.058$ & $19.277 \pm 0.673 \#$ & $1.382 \pm 0.076$ & $1.388 \pm 0.096$ \\
Rot+Ant & 6 & $42.818 \pm 0.694$ & $32.980 \pm 1.119 \#$ & $1.791 \pm 0.072$ & $1.876 \pm 0.121$ \\
DPI+TTFA & 6 & $28.630 \pm 0.332$ & $16.709 \pm 1.089 \#$ & $0.873 \pm 0.072$ & $0.917 \pm 0.052$ \\
DPI+Ant & 6 & $41.486 \pm 3.265$ & $35.683 \pm 0.440 \#$ & $1.197 \pm 0.098$ & $1.222 \pm 0.118$ \\
TTFA+Ant & 6 & $47.715 \pm 2.015$ & $28.617 \pm 0.520^{\#}$ & $1.665 \pm 0.082$ & $1.609 \pm 0.082$ \\
\hline
\end{tabular}

Table 8. Effect of GSH on electron leakage from mitochondrial $\mathrm{FADH}_{2}$ respiratory chain induced by $\mathrm{Cr}(\mathrm{VI})$. Note: ${ }^{*} P<0.05$ vs the $\mathrm{Cr}(\mathrm{VI})$ group. The concentration of $\mathrm{Cr}(\mathrm{VI}), \mathrm{GSH}$, Rot, DPI , TTFA and Ant were $50 \mu \mathrm{M}$, $200 \mu \mathrm{M}, 6.35 \mu \mathrm{M}, 10 \mu \mathrm{M}, 20 \mu \mathrm{M}$ and $3.75 \mu \mathrm{M}$, respectively.

\section{Effect of GSH on electron leakage from liver mitochondrial respiratory chain}

Effect of GSH on the NADH and $\mathrm{FADH}_{2}$ respiratory chains in liver mitochondria were shown in Figure 7. ROS production in the $\mathrm{Cr}(\mathrm{VI})$ treatment groups were significantly higher than that in groups treated with $\mathrm{Cr}(\mathrm{VI})+\mathrm{GSH}(P<0.05)$ (Table 7). However, no significant difference in electron leakage was observed between groups treated with $\mathrm{Cr}(\mathrm{VI})$ alone and groups treated with $\mathrm{Cr}(\mathrm{VI})+\mathrm{GSH}$ (Table 8).

\section{Discussion}

Mitochondria, rich in hepatocytes, provide the main source of cytosolic ATP via the oxidative phosphorylation system. As is well known, $\operatorname{Cr}(\mathrm{VI})$ could move into hepatocyte by non-specific ion channels and produce large amounts of ROS, which would damage mitochondrial membrane structure and energy metabolism, and further induce cellular apoptosis [11]. Generally, the quantity of ROS generated varies under different sites, substrates and mitochondrial metabolic conditions caused by different chemicals [12]. Many studies reported that critical sites for electron leakage were the ubiquinone binding sites of complex I and complex III, and that of complex II were negligible [13, 14]. Some studies also suggest that ROS would be produced from complex II and complex IV $[15,16]$. However, the mechanism of how excess ROS is produced in hepatic mitochondria exposed to $\mathrm{Cr}(\mathrm{VI})$ has not been elaborated clearly.

Above all, we separated mitochondria from the rat liver by differential centrifugation and ensured mitochondria could maintain integrity and functionality at a suitable level during experiments. Then we used several inhibitors of complexes to characterize the mechanism of enhanced ROS production in hepatic mitochondria treated with $\mathrm{Cr}(\mathrm{VI})$. Rot, which is highly lipophilic, could easily penetrate the membrane and selectively block electrons transferring from iron-sulfur center of complex I to ubiquinone [17]. DPI, as FMN site-specific inhibitor of complex I, could combine flavin mononucleotide to reduce electron leakage from FMN site of complex I [18]. The main effect of TTFA is to inhibit electron transfer from complex II ubiquinone binding site to complex III [19]. Ant, the complex III inhibitor, acts on complex III and inhibit electron driving from cytochrome $\mathrm{b}(\mathrm{Cyt} \mathrm{bH})$ to ubiquinone, which could increase electron leakage and ROS production [20,21].

ROS production in succinate-energized hepatic mitochondria treated with $\mathrm{Cr}(\mathrm{VI})$ were much higher than that in glutamate/malate-energized mitochondria, which may due to 


\section{Cellular Physiology and Biochemistry}

Cell Physiol Biochem 2013;31:473-485

\begin{tabular}{l|l}
\hline DOI: $10.1159 / 000350062$ & (C) 2013 S. Karger AG, Basel
\end{tabular}

higher basal respiratory rate of $\mathrm{FADH}_{2}$ respiratory chain. In glutamate/malate-energized mitochondria, ROS production increased in the Rot group. It is suggested that in mitochondria under normal physiological conditions, Rot inhibit electron driving from iron-sulfur center of complex I to ubiquinone and enhance their leakage from NADH respiratory chain [11]. ROS generation decreased in the DPI group, indicated that DPI would act on FMN site and block electron moving into the reduction center of complex I and then decrease electron leakage. These results were similar with others' studies [22]. However, when mitochondria were treated with $\mathrm{Cr}(\mathrm{VI})$, the results were different. ROS content and electron leakage increased in the Rot $+\mathrm{Cr}(\mathrm{VI})$ group, but not changed in the DPI+Cr(VI) group. The results suggested that the ubiquinone binding site, but not the FMN site, is the main site of ROS production in NADH respiratory chain. Moreover, Ant could increase ROS production and electron leakage in mitochondria treated with $\mathrm{Cr}(\mathrm{VI})$, which suggests that Ant could inhibit electron moving from Cyt bH to ubiquinone, accumulate semiquinone and increase electron leakage [23, 24].

With regard to mitochondrial $\mathrm{FADH}_{2}$ respiratory chain, it is generally acknowledged that complex II could not release electron by itself and electron could leak from complex I when they move backward from complex II to complex I [25], or leak from the ubiquinone binding site of complex III if they move forward along respiratory chain [26]. In succinate-energized mitochondria without $\mathrm{Cr}(\mathrm{VI})$ treatment, ROS production and electron leakage decreased in the Rot group and DPI group, and increased in the TTFA group and the Ant group. It is suggested that when electron generated from succinate moves backward from complex II to complex I, Rot would inhibit this process and DPI protects the FMN site of complex I to reduce electron leakage [18]. On the contrary, TTFA could block electron moving backward from complex II to ubiquinone, while Ant acts on the ubiquinone binding site of complex III, which leads to electron leakage and excess ROS generation.

As far as succinate-energized mitochondria treated with $\mathrm{Cr}(\mathrm{VI})$ are concerned, compared with the $\mathrm{Cr}(\mathrm{VI})$ group, ROS content and electron leakage in the Rot $+\mathrm{Cr}(\mathrm{VI})$ group and the DPI+ $\mathrm{Cr}(\mathrm{VI})$ group showed no significant difference. ROS generation in $\mathrm{Cr}(\mathrm{VI})$-treated mitochondria was irrelevant with electron moving backward from complex II to complex I. Then ROS content and electron leakage increased in the $\mathrm{Cr}(\mathrm{VI})+\mathrm{TTFA}$ group compared with the $\mathrm{Cr}(\mathrm{VI})$ group. It is seemingly found that co-treatment of $\mathrm{Cr}(\mathrm{VI})$ and TTFA could promote electron leaked from the ubiquinone binding site of complex II, however, ROS content and electron leakage in the $\operatorname{Cr}(\mathrm{VI})+$ Rot+TTFA group and the $\operatorname{Cr}(\mathrm{VI})+\mathrm{DPI}+\mathrm{TTFA}$ group were significantly lower than those in the $\mathrm{Cr}(\mathrm{VI})+$ TTFA group. It is rational to postulate that the increase of electron leakage in the $\mathrm{Cr}(\mathrm{VI})+\mathrm{TTFA}$ group was actually due to the backflow of electron from complex II to complex I. Furthermore, after Ant inhibits the ubiquinone binding site of complex III in mitochondria exposed to $\mathrm{Cr}(\mathrm{VI})$, ROS content and electron leakage increased significantly. The result above suggested that $\mathrm{Cr}(\mathrm{VI})$ could induce electron leakage from $\mathrm{FADH}_{2}$ respiratory chain in liver mitochondria through the ubiquinone binding site of complex III, other than enhancing electron backflow from complex II to complex I or leaking from the ubiquinone binding site of complex II .

GSH, a critical endogenous antioxidant in mitochondria, could transform $\mathrm{H}_{2} \mathrm{O}_{2}$ into $\mathrm{H}_{2} \mathrm{O}$ under the effect of glutathione peroxidase and transform itself into oxidized glutathione disulfide (GSSG), which could then transform into glutathione by glutathione reductase. Excess $\mathrm{H}_{2} \mathrm{O}_{2}$ could be cleared during this cycle [27]. Cederbaum et al. found that exogenous S-adenosyl-L-methionine (SAM) supplement, as a precursor of GSH synthesis, could protect liver mitochondria from oxidative damage $[28,29]$. In our study, protective effects of exogenous GSH supplement on NADH and $\mathrm{FADH}_{2}$ respiratory chain of liver mitochondria were similar. GSH could clear over excess ROS production in liver mitochondria induced by $\mathrm{Cr}(\mathrm{VI})$ in both $\mathrm{NADH}$ and $\mathrm{FADH}_{2}$ respiratory chains, but no significant protective effect could be observed on electron leakage. It is indicated that GSH do exhibit protective effects on liver mitochondria by the elimination of excess $\mathrm{H}_{2} \mathrm{O}_{2}$ and ROS, but could not decrease electron leakage from mitochondrial respiratory chain.

In summary, our study demonstrated the sites of $\mathrm{Cr}(\mathrm{VI})$-induced ROS production and the protective effect of GSH in mitochondrial $\mathrm{NADH}$ and $\mathrm{FADH}_{2}$ respiratory chain. $\mathrm{Cr}(\mathrm{VI})$ 


\section{Cellular Physiology and Biochemistry}

Cell Physiol Biochem 2013;31:473-485

DOI: $10.1159 / 000350062$

Published onIIne: IVarch 20, 2013

(c) 2013 S. Karger AG, Basel

www.karger.com/cpb

induced the increase of electron leakage from ubiquinone binding sites of complex I and complex III in NADH respiratory chain. While in succinate-energized mitochondria, $\mathrm{Cr}(\mathrm{VI})$ induced electron leakage from the ubiquinone binding site of complex III when the electron moved forward in the respiratory chain. GSH can alleviate $\mathrm{Cr}(\mathrm{VI})$-induced liver mitochondrial oxidative damage through effective removal of excess $\mathrm{H}_{2} \mathrm{O}_{2}$ and total ROS content, rather than the improvement of mitochondrial respiration function to reduce electron leakage.

\section{Acknowledgements}

We are very grateful for the generous assistance of Physiology Department and Preventive Medicine Experimental Center of Central South University, Changsha, China. This work was supported by the National Natural Science Foundation of China (No. 30972511). The authors declare no conflict of interest.

\section{References}

1 Mohan D, Pittman CU Jr: Activated carbons and low cost adsorbents for remediation of tri- and hexavalent chromium from water. J Hazard Mater 2006;137:762-811.

-2 Wang XF, Xing ML, Shen Y, Zhu X, Xu LH: Oral administration of Cr (VI) induced oxidative stress, DNA damage and apoptotic cell death in mice. Toxicology 2006;228:16-23.

3 Hoffman DL, Brookes PS: Oxygen sensitivity of mitochondrial reactive oxygen species generation depends on metabolic conditions. J Biol Chem 2009;284:16236-16245.

4 Li J, Xu Z, Tan M, Su W, Gong XG: 3-(4-(Benzo[d] thiazol-2-yl) -1-phenyl- 1H- pyrazol-3-yl) phenyl acetate induced Hep G2 cell apoptosis through a ROS-mediated pathway. Chem Biol Interact 2010;183:341-348.

5 Murphy MP: How mitochondria produce reactive oxygen species. Biochem J 2009;417:1-13.

6 Gopi S, Setty OH: Protective effect of Phyllanthus fraternus against bromobenzene induced mitochondrial dysfunction in rat liver mitochondria. Food Chem Toxicol 2010;48:2170-2175.

-7 Fernandez-Checa JC, Kaplowitz N: Hepatic mitochondrial glutathione: transport and role in disease and toxicity. Toxicol Appl Pharmacol 2005;204:263-273.

-8 Sailaja R, Setty OH: Protective effect of Phyllanthus fraternus against allyl alcohol-induced oxidative stress in liver mitochondria. J Ethnopharmacol 2006;105:201-209.

-9 Wen JJ, Garg NJ: Mitochondrial generation of reactive oxygen species is enhanced at the Q(o) site of the complex III in the myocardium of Trypanosoma cruzi-infected mice: beneficial effects of an antioxidant. J Bioenerg Biomembr 2008;40:587-598.

-10 Fernández-Vizarra E, Ferrín G, Pérez-Martos A, Fernández-Silva P, Zeviani M, Enríquez JA: Isolation of mitochondria for biogenetical studies: An update. Mitochondrion 2010;10:253-262.

-11 Panieri E, Gogvadze V, Norberg E, Venkatesh R, Orrenius S, Zhivotovsky B: Reactive oxygen species generated in different compartments induce cell death, survival, or senescence. Free Radic Biol Med DOI: 10.1016/j.freeradbiomed.2012.12.024.

-12 Hoffman DL, Brookes PS: Oxygen sensitvity of mitochondrial reactive oxygen species generation depends on metabolic conditions. J Biol Chem 2009;284:16236-16245.

-13 Viola HM, Hool LC: Qo site of mitochondrial complex III is the source of increased superoxide after transient exposure to hydrogen peroxide. J Mol Cell Cardiol 2010;49:875-885.

14 Brand MD: The sites and topology of mitochondrial superoxide production. Exp Gerontol 2010;45:466472.

15 Zhang JG, Tirmenstein MA, Nicholls-Grzemski FA, Fariss MW: Mitochondrial electron transport inhibitors cause lipid peroxidation-depedent and independent cell death: protective role of antioxidants. Arch Biochem Biophy 2002;393:87-96. 


\section{Cellular Physiology and Biochemistry}

Cell Physiol Biochem 2013;31:473-485

\begin{tabular}{l|l}
\hline DOI: $10.1159 / 000350062$ & (c) 2013 S. Karger AG, Basel
\end{tabular}

\16 Moreno-Sánchez R, Hernández-Esquivel L, Rivero-Segura NA, Marín-Hernández A, Neuzil J, Ralph SJ, Rodríguez-Enríquez S: Reactive oxygen species are generated by the respiratory complex II - evidence for lack of contribution of the reverse electron flow in complex I. FEBS J 2013;280:927-938.

17 Fato R, Bergamini C, Leoni S, Lenaz G: Mitochondrial production of reactive oxygen species: role of complex I and quinone analogues. Biofactors 2008;32:31-39.

18 Lambert AJ, Buckingham JA, Boysen HM, Brand MD: Diphenyleneiodonium acutely inhibits reactive oxygen species production by mitochondrial complex I during reverse, but not forword electron transport. Biochim Biophys Acta 2008;1777:397-403.

19 Zhang JG, Nicholls-Grzemski FA, Tirmenstein MA, Fariss MW: Vitamin E succinate protects hepatocytes against the toxic effect of reactive oxygen species generated at mitochondrial complexes I and III by alkylating agents. Chem Biol Interact 2001;138:267-284.

20 Liang B, Wang L, He T, Liu W, Li Q Li M: In vitro reactive oxygen species production by mitochondria from the rabbitfish Siganus fuscessens livers and the effects of Irgarol-1051. Environ Toxicol Pharmacol 2012;35:154-160.

-21 Shiryaeva A, Arkadyeva A, Emelyanova L, Sakuta G, Morozov V: Superoxide anion production by the mitochondrial respiratory chain of hepatocytes of rats with experimental toxic hepatitis. J Bioenerg Biomembr 2009;41:379-385.

22 Fato R, Bergamini C, Bortolus M, Maniero AL, Leoni S, Ohnishi T, Lenaz G: Differential effects of mitochondrial Complex I inhibitors on production of reactive oxygen species. Biochim Biophys Acta 2009;1787:384-392.

23 Fang J, Beattie DS: Rotenone-insensitive NADH-dehydrogenase is a potential source of superoxide in procyclic Try panosoma brucei mitochondria. Mol Biochem Parasitol 2002;123:135-142.

24 Dröse S, Hanley PJ, Brandt U: Ambivalent effect of diazoxide on mitochodrial ROS production at respiratory chain complexes I and III. Biochim Biophys Acta 2009;1790:558-565.

25 Han D, Canali R, Rettori D, Kaplowitz N: Effect of Glutathione Depletion on Sites and Topology of Superoxide and Hydrogen Peroxide Production in Mitochondria. Mol Pharmacol 2003;64:1136-1144.

-26 Liu Y, Fiskum G, Schubert D: Generation of reactive oxygen species by the mitochondrial electron transport chain. J Neurochem 2002;80:780-787.

27 Yuan L, Kaplowitz N: Glutathione in liver diseases and hepatotoxicity. Mol Aspects Med 2009;30:29-41.

28 Cederbaum AI: Hepatoprotective effects of S-adenosyl-L-methionine against alcohol- and cytochrome P450 2E1-induced liver injury. World J Gastroenterol 2010;16:1366-1376.

29 Wang X, Cederbaum AI: S-adenosyl-L-methionine decreases the elevate Fas agonistic antibody plus acute ethanol pretreatment in mice. Arch Biochem Biophys 2008;477:1-11. 